\author{
Ecología
}

\title{
Diversidad de Ichneumonidae (Hymenoptera) en un bosque de Pinus spp. y Juniperus flaccida en Jaumave, Tamaulipas, México
}
Diversity of Ichneumonidae (Hymenoptera) in a Pinus spp. and Juniperus flaccida forest in Jaumave, Tamaulipas, Mexico

\author{
Arely Julieta Rodríguez-Mota ${ }^{a}$, Enrique Ruíz-Cancino ${ }^{\mathrm{a}, *}$, Andrey Ivanovich-Khalaima, ${ }^{\mathrm{a}, \mathrm{b}}$, \\ Juana María Coronado-Blanco ${ }^{\mathrm{a}}$ y Jacinto Treviño-Carreón ${ }^{\mathrm{a}}$ \\ ${ }^{a}$ Facultad de Ingeniería y Ciencias, Universidad Autónoma de Tamaulipas, Centro Universitario Adolfo López Mateos, 87149 Ciudad Victoria, Tamaulipas, México \\ ${ }^{\mathrm{b}}$ Instituto Zoológico, Academia de Ciencias de Rusia, University Embankment 1, 199034 San Petersburgo, Rusia
}

Recibido el 2 de marzo de 2015; aceptado el 2 de junio de 2015

Disponible en Internet el 21 de octubre de 2015

\begin{abstract}
Resumen
Los icneumónidos son avispas parasitoides de importancia en el control biológico de insectos plaga y constituyen una de las familias de Hymenoptera con mayor diversidad. El objetivo de esta investigación fue conocer la diversidad de Ichneumonidae y la relación con la temperatura ambiental y la precipitación pluvial en una localidad de Jaumave, Tamaulipas, México, durante un año. Se colocó una trampa Malaise a 1,450 m snm en una asociación de Pinus nelsonii, P. cembroides y Juniperus flaccida en una área seca, aunque con una pequeña corriente de agua cerca. Se recolectaron 1,115 icneumónidos, de los cuales la subfamilia Cryptinae fue la mejor representada en número de géneros y especies, siendo Pimpla el género más abundante y Pachysomoides stupidus la especie con más individuos. Se encontró una gran diversidad de ejemplares correspondientes a la familia estudiada. La temperatura presentó una correlación alta con la diversidad de Ichneumonidae, la precipitación pluvial una correlación moderada. Tres especies son nuevos registros para México y 5 especies para Tamaulipas, por lo que se conocen 1,298 especies de Ichneumonidae para el país. Este trabajo contribuye al conocimiento de Ichneumonidae en áreas secas de México.

Derechos Reservados (C) 2015 Universidad Nacional Autónoma de México, Instituto de Biología. Este es un artículo de acceso abierto distribuido bajo los términos de la Licencia Creative Commons CC BY-NC-ND 4.0.
\end{abstract}

Palabras clave: Avispas parasitoides; Ichneumonoidea; Trampa Malaise; Riqueza de especies; Temperatura; Precipitación pluvial

\section{Abstract}

Ichneumonids are parasitoid wasps with importance in the biological control of insect pests and constitute one of the most diverse families of Hymenoptera. The objective of this research was to know the diversity of Ichneumonidae and its relationship with temperature and rainfall in one locality of Jaumave, Tamaulipas, Mexico, during one year. One Malaise tramp was operated at 1,450 m in a dry forest with Pinus nelsonii, P. cembroides and Juniperus flaccida, with a small source of water near it. The number of ichneumonids collected was 1,115 . Cryptinae was the subfamily best represented in number of genera and species, Pimpla the genus more abundant and Pachysomoides stupidus the species with more individuals. High diversity of this family was found in the studied locality. High correlation was found between Ichneumonidae and temperature, and a moderate correlation with rainfall. Three species are new records for Mexico and 5 for Tamaulipas, therefore 1,298 species are known in the country. This research contributes to the knowledge of Ichneumonidae in dry areas from Mexico.

All Rights Reserved (C) 2015 Universidad Nacional Autónoma de México, Instituto de Biología. This is an open access item distributed under the Creative Commons CC License BY-NC-ND 4.0.

Keywords: Parasitoids; Ichneumonoidea; Malaise trap; Species richness; Temperature; Rainfall

\footnotetext{
* Autor para correspondencia.

Correo electrónico: eruiz@uat.edu.mx (E. Ruíz-Cancino).

La revisión por pares es responsabilidad de la Universidad Nacional Autónoma de México.
} 


\section{Introducción}

Dentro del orden Hymenoptera, las familias Ichneumonidae y Braconidae forman la superfamilia Ichneumonoidea (Gauld y Bolton, 1988), una de las superfamilias de avispas parasitoides de mayor importancia en el control biológico de insectos plaga (Nicholls, 2008). Los himenópteros parasitoides son considerados el grupo más rentable de todos los organismos utilizados en el control biológico de insectos plaga (Anento y Selfa, 1997). Las Ichneumonidae son una de las familias con mayor número de especies de Hymenoptera (Ruíz, 2010) y están distribuidas en todas las regiones zoogeográficas del mundo (Gauld, 2006). La mayor diversidad de especies se presenta en zonas con climas templados (Gauld y Bolton, 1988). Whittaker (1960) fue uno de los primeros autores en mencionar el término «diversidad alfa» para referirse a la riqueza en especies de una muestra territorial; Halffer y Moreno (2005) dividen el concepto de diversidad alfa en 3 puntos: a) puntual: número de especies que tiene una comunidad en un punto determinado; b) promedio: un promedio de valores puntuales correspondientes a diferentes lugares de un paisaje ocupado por una misma comunidad, y c) acumulada: número de especies que se recolecta en un punto determinado en un cierto lapso de tiempo. Para esta investigación, el concepto más adecuado es el de diversidad alfa acumulada. Para la zona de estudio no se contaba con un registro de las especies de Ichneumonidae; por lo tanto, se planteó el objetivo de evaluar la diversidad de Ichneumonidae y su relación con la temperatura ambiental y precipitación pluvial en una localidad de Jaumave, Tamaulipas, México, durante un año.

\section{Materiales y métodos}

El estudio se llevó a cabo en el ejido Magdaleno Aguilar, municipio de Jaumave, Tamaulipas, México. El clima es semicálido subhúmedo con lluvias en verano $\mathrm{A}(\mathrm{C}) \mathrm{w}_{0}$ y el tipo de vegetación de la zona es una asociación de pinos (Pinus nelsonii Shaw y P. cembroides Zucc.) y cedro blanco o junípero (Juniperus flaccida Schltdl.); en la vegetación herbácea y arbustiva se encuentran otras 44 especies. En el sitio hay una muy pequeña corriente de agua permanente. Se colocó una trampa Malaise elaborada de malla, que se emplea principalmente para recolectar insectos voladores, localizada a $23^{\circ} 26^{\prime} 59.7^{\prime \prime} \mathrm{N}$ y $99^{\circ} 33^{\prime} 26.4^{\prime \prime} \mathrm{O}$ a 1,450 m snm. Las colectas de las avispas Ichneumonidae se efectuaron durante un año, entre septiembre del 2011 y agosto del 2012. La temperatura ambiental media fue de $18.8^{\circ} \mathrm{C}$ (máxima, 38 ; mínima $0{ }^{\circ} \mathrm{C}$ ) y la precipitación pluvial fue de $688.8 \mathrm{~mm}$ acumulados durante el año de estudio; estos datos se obtuvieron de la estación meteorológica Magdaleno Aguilar, región hidrológica No. 26, cuenca del río Guayalejo, ubicada en los $23^{\circ} 27^{\prime} 13^{\prime \prime} \mathrm{N}, 99^{\circ} 30^{\prime} 33^{\prime \prime} \mathrm{O}$, a 1,430 m snm.

Los insectos se montaron en alfileres entomológicos o en triángulos de papel opalina holandesa, según su tamaño. Para la determinación de los ejemplares se utilizaron claves taxonómicas y descripciones originales de las subfamilias, géneros y especies de Gauld (1991, Pimplinae); Gauld, Wahl, Bradshaw, Hanson y Ward (1997,
Anomaloninae, Diplazontinae, Tryphoninae); Gauld, Ward y Mallet (2000, Brachycyrtinae); Gauld, Godoy, Sithole y Ugalde (2002, Banchinae y Metopiinae); Kasparyan y Ruíz (2005 y 2008, Cryptinae); Khalaim y Ruíz-Cancino (2012, Banchinae: Exetastes); Townes (1969, Banchinae, Ichneumoninae; Alomyinae: Centeterus); Townes (1970a, Cryptinae); Townes (1970b, Banchinae, Campopleginae); Townes (1971, Diplazontinae, Mesochorinae, Metopiinae, Ophioninae, Orthocentrinae, Tersilochinae), Townes y Townes (1959, Metopiinae); Townes y Townes (1966, subfamilias y géneros neotropicales), y Townes y Townes (1978, Banchinae). Para conocer la distribución geográfica de las especies se usó principalmente la base de datos de Yu, van Achterberg y Horstman (2012). El material se depositó en el museo de insectos de la Facultad de Ingeniería y Ciencia-UAT, en Ciudad Victoria, Tamaulipas.

Para evaluar la diversidad alfa a través del tiempo se utilizaron 2 índices basados en la cuantificación del número de especies presentes que indican la riqueza específica: el índice de Margalef $\left(D_{M g}\right)$ y el índice de Menhinick $\left(D_{M n}\right)$. Estos suponen que existe una relación entre el número de especies y el número de individuos (Moreno, 2001). En el índice de Margalef los valores superiores a 5 indican biodiversidad alta y los valores inferiores a 2, biodiversidad baja. En el de Menhinick, los valores de 3 en adelante indican zonas de biodiversidad alta. Las fórmulas para calcular los índices son:

$$
\mathrm{D}_{\mathrm{Mg}}=\mathrm{s}-1 / \operatorname{Ln}(\mathrm{n}) \text { y } \mathrm{D}_{\mathrm{Mn}}=\mathrm{s} / \sqrt{\mathrm{n}}
$$

donde $s=$ número de especies y $n=$ número total de individuos en la muestra.

Se llevó a cabo un análisis de correlación entre los resultados de los índices de diversidad obtenidos y los registros de temperatura y precipitación pluvial del área de estudio para conocer el grado de relación o asociación entre las variables (Hopkins, Hopkins y Glass, 1997) con ayuda del programa Statistics. Además, se calculó la riqueza de especies para cada subfamilia de Ichneumonidae.

\section{Resultados}

Se recolectaron 1,115 icneumónidos, pertenecientes a 17 subfamilias, 86 géneros y 59 especies; además, se separó material de otras 110 morfoespecies. En el Anexo 1 se presentan las subfamilias, géneros y especies obtenidos por mes de recolecta, así como el número de hembras y machos. Las Pimplinae, Campopleginae y Cryptinae fueron las más abundantes en cuanto al número de individuos recolectados, géneros y especies; Ichneumoninae destacan solo en el número de géneros (17). Se recolectaron 591 hembras y 524 machos. De las 17 subfamilias, 9 estuvieron representadas solo por un género: Alomyinae, Anomaloninae, Brachycyrtinae, Diplazontinae, Mesochorinae, Nonninae, Ophioninae, Poemeniinae y Tryphoninae.

En mayo se recolectó el mayor número de ejemplares (254) y en diciembre, el menor (11). Los géneros con mayor número de ejemplares fueron Pimpla Fabricius (267), Diadegma Förster (124), Orthocentrus Gravenhorst (72) e Hyposoter Förster (60). El resto de los géneros recolectados presentaron menos de 
Tabla 1

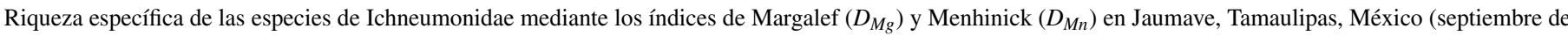
2011-agosto de 2012).

\begin{tabular}{|c|c|c|c|c|c|c|c|c|c|c|c|c|}
\hline & \multicolumn{12}{|c|}{ Meses } \\
\hline & sep & oct & nov & dic & ene & feb & mar & abr & may & jun & jul & ago \\
\hline$D_{M g}$ & 6.14 & 8.52 & 5.02 & 2.50 & 3.91 & 3.03 & 6.21 & 9.05 & 11.20 & 10.29 & 9.79 & 13.69 \\
\hline$D_{M n}$ & 3.83 & 4.76 & 3.17 & 2.11 & 2.50 & 2.41 & 3.47 & 3.70 & 3.95 & 4.49 & 4.10 & 5.38 \\
\hline
\end{tabular}

34 individuos, e incluso una gran cantidad de géneros estuvieron representados solo por 1-2 ejemplares. Con respecto a las especies, las más abundantes fueron Pachysomoides stupidus (Cresson) (31), Meniscomorpha romata Ugalde y Gauld (21), Exetastes pictus Cushman (20) y Zaglyptus simonis (Marshall) (18), además de 2 especies sin determinar de Diadegma (32 y 23) y una de Hyposoter (21). Los nuevos registros para México son Diapetimorpha rufigaster Cushman, Dichrogaster gemina Townes y Poemenia albipes (Cresson). Además, Calliephialtes thurberiae Cushman y el género Leptobatopsis Ashmead representan nuevos registros del estado de Tamaulipas.

La zona de estudio pertenece a la región Neártica. Las relaciones zoogeográficas de las especies son: 10.2\% Neártica (6 especies), 86.4\% Neártica y Neotropical (51), 1.7\% Neártica y Oceánica (1) y $1.7 \%$ Holártica (1).

Los índices de diversidad presentaron valores que indican que la zona es de biodiversidad alta para Ichneumonidae, siendo de marzo a agosto los meses con mayor diversidad, además de los primeros 3 meses de estudio: septiembre-noviembre; agosto obtuvo los valores más altos para los índices de Margalef y Menhinick, con 13.69 y 5.38, respectivamente. Esta situación pudo estar influida por la presencia de 47 especies vegetales en el sitio y por los insectos fitófagos que se alimentan de ellas, los cuales son los huéspedes de Ichneumonidae. Diciembre, enero y febrero fueron los meses con menor diversidad (tabla 1). En otra investigación efectuada en Tamaulipas con Ichneumonidae en una trampa Malaise por un año, en un matorral espinoso cercano al río San Marcos, Victoria, el cual está bordeado por un bosque de galería, el índice de Margalef fue de 19.31 (Pérez-Urbina et al., 2010). Por su parte, en San Pedro, Perú, a 1,500 m snm, se obtuvo un índice de Margalef de 11.99 al estudiar la subfamilia Rogadinae de Braconidae (Sulca-Garro y Castillo, 2013).

En el cálculo de la riqueza específica por subfamilias, Cryptinae y Campopleginae fueron las que presentaron los valores más altos para ambos índices de diversidad. Para el caso específico del índice de Margalef, 5 subfamilias (Brachycyrtinae, Diplazontinae, Nonninae, Tersilochinae y Tryphoninae) mostraron valores igual a 0 en el dividendo; esto se debió a que solo se determinó una especie. Además, 2 subfamilias (Anomaloninae y Poemeniinae) estuvieron representadas por un individuo (tabla 2).

En las figuras 1 y 2 se representan la temperatura y la precipitación pluvial ocurridas entre septiembre de 2011 y agosto de 2012; cabe mencionar que a pesar de que la zona muestra un tipo de clima con lluvias en verano, ocurrió un evento atípico de precipitación pluvial en invierno. Sobre la distribución anual de individuos y de géneros y/o especies recolectados, la mayor cantidad se concentró desde abril hasta agosto, en donde
Tabla 2

Riqueza específica de las subfamilias de Ichneumonidae mediante los índices de Margalef $\left(D_{M g}\right)$ y Menhinick $\left(D_{M n}\right)$ en Jaumave, Tamaulipas, México (septiembre de 2011-agosto de 2012).

\begin{tabular}{lll}
\hline Subfamilia & $D_{M g}$ & $D_{M n}$ \\
\hline Alomyinae & 0.96 & 1.06 \\
Anomaloninae & $\mathrm{E}$ & 1.00 \\
Banchinae & 3.71 & 2.12 \\
Brachycyrtinae & 0 & 0.58 \\
Campopleginae & 7.38 & 2.50 \\
Cryptinae & 9.24 & 3.29 \\
Diplazontinae & 0 & 0.71 \\
Ichneumoninae & 2.84 & 1.58 \\
Mesochorinae & 1.30 & 1.26 \\
Metopiinae & 0.72 & 0.75 \\
Nonninae & 0 & 0.58 \\
Ophioninae & 1.44 & 1.41 \\
Orthocentrinae & 0.23 & 0.23 \\
Pimplinae & 3.30 & 1.12 \\
Poemeniinae & $\mathrm{E}$ & 1.00 \\
Tersilochinae & 0 & 0.71 \\
Tryphoninae & 0 & 0.58 \\
\hline E: error porqu & &
\end{tabular}

E: error porque la fórmula divide entre cero.

el incremento empieza a ser notorio desde marzo, con un rango de precipitación pluvial de 29.5-143.5 mm, precedido por un pico de precipitación en febrero de $92.5 \mathrm{~mm}$. En relación con la temperatura ambiental, la mayor cantidad de capturas sucedió entre los $17.6-21.8^{\circ} \mathrm{C}$ de temperatura mensual media.

En todos los análisis de correlación que se desarrollaron se encontró una relación positiva o ascendente. Entre la precipitación pluvial (variable independiente) y la diversidad de icneumónidos (variable dependiente) existe una correlación

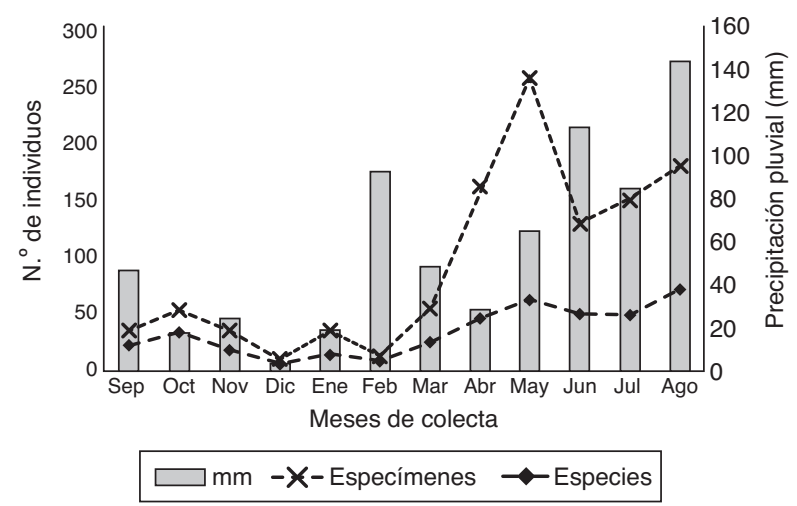

Figura 1. Distribución anual del número de ejemplares y riqueza de las especies de Ichneumonidae en función de la precipitación pluvial en bosque de Pinus spp. y Juniperus flaccida en Jaumave, Tamaulipas, México (septiembre de 2011 agosto de 2012). 


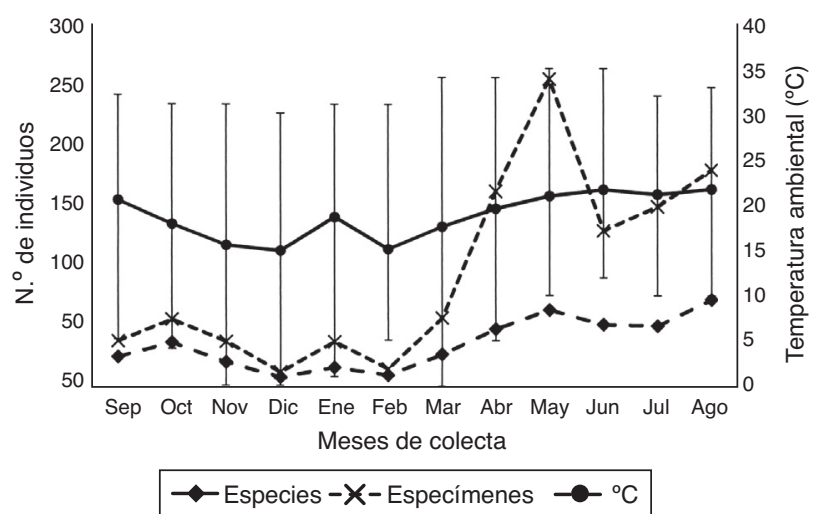

Figura 2. Distribución anual del número de ejemplares y riqueza de las especies de Ichneumonidae en función de la temperatura ambiental en bosque de Pinus spp. y Juniperus flaccida en Jaumave, Tamaulipas, México (septiembre de 2011agosto de 2012).

moderada. Con el índice de Margalef, el coeficiente de correlación $(R)$ es igual a $0.6303(p=0.0006)$ y con un coeficiente de determinación $\left(R^{2}\right)$ es igual a 0.3973 , con lo cual se indica que la precipitación pluvial explica el $39.73 \%$ de la diversidad (fig. 3a). Para el índice de Menhinick, $R=0.5606(p=0.0003)$ y $R^{2}=0.3143$, la precipitación pluvial explica el $31.43 \%$ de la diversidad (fig. 3b). Para los análisis entre la temperatura ambiental (variable independiente) y la diversidad (variable dependiente) existe una correlación alta; con el índice de Margalef, $R=0.8483$ y $R^{2}=0.7197$, la temperatura ambiental explica el $71.97 \%$ de la diversidad observada (fig. 4a). Para el índice de Menhinick, $R=0.7549$ y $R^{2}=0.5699$, la temperatura ambiental explica el $56.99 \%$ de la diversidad (fig. 4b).
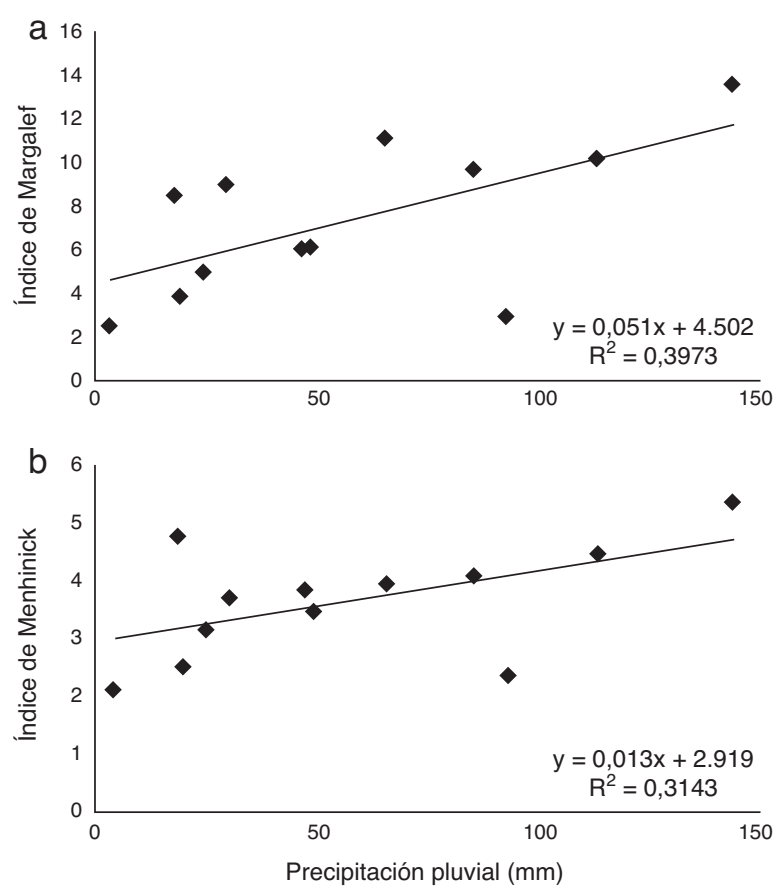

Figura 3. Regresión lineal simple entre la precipitación pluvial y los valores de los índices de diversidad de Ichneumonidae en bosque de Pinus spp. y Juniperus flaccida en Jaumave, Tamaulipas, México (septiembre de 2011-agosto de 2012). a, Índice de Margalef; b, índice de Menhinick.
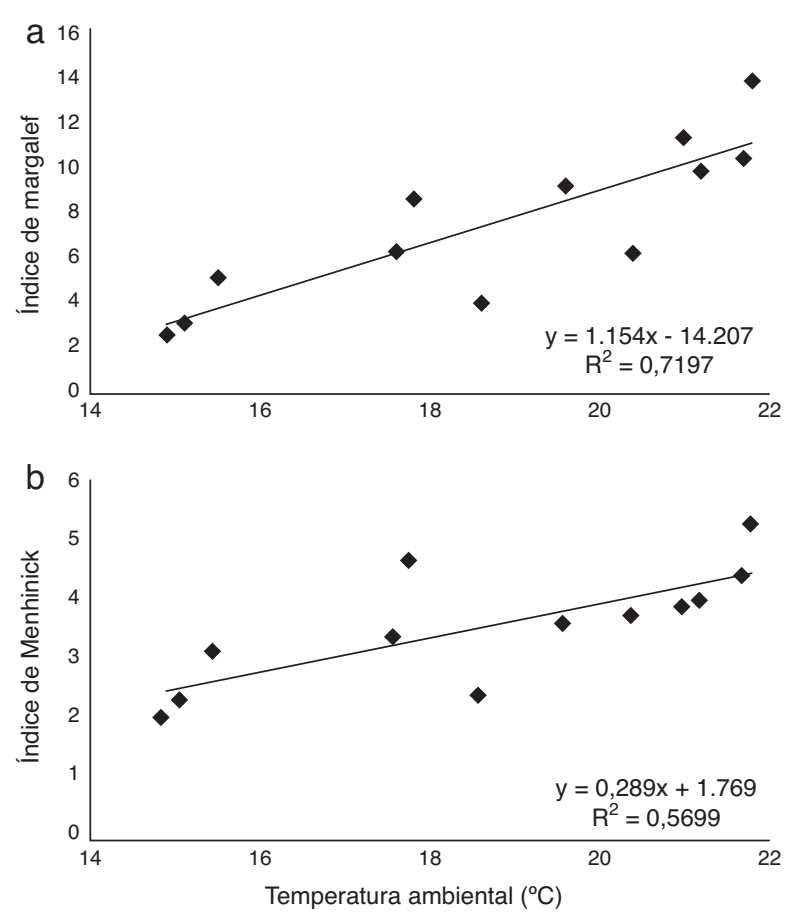

Figura 4. Regresión lineal simple entre la temperatura ambiental y los valores de los índices de diversidad de Ichneumonidae en bosque de Pinus spp. y Juniperus flaccida en Jaumave, Tamaulipas, México (septiembre de 2011-agosto de 2012). a, Índice de Margalef; b, índice de Menhinick.

\section{Discusión}

Las subfamilias más abundantes fueron Campopleginae, Cryptinae y Pimplinae, así como Ichneumoninae por ser abundante en géneros; la misma situación documentaron PérezUrbina et al. (2010) en el Cañón del Novillo, otra localidad del estado de Tamaulipas. En dicho estudio, Cryptinae aparece como la subfamilia más diversa según el índice de Margalef, lo cual también se registra en el presente estudio (tabla 2). La diferencia entre ambos estudios resultó entre los géneros con más individuos y en la especie más abundante de las determinadas, siendo para el presente estudio Pachysomoides stupidus, y la documentada por Pérez-Urbina et al. (2010) fue Pimpla sanguinipes Cresson.

La mayor cantidad de individuos y especies recolectados coincidió con el inicio de la primavera, antecedido por un pico de precipitación pluvial (fig. 3). Además, la distribución anual de las especies es relativamente paralela a la temperatura ambiental media (fig. 4); resultados similares registraron Falcó, Oltra, Moreno, Pujade-Villar y Jiménez (2006) en el Pirineo de Andorra para Braconidae. La mayor diversidad de Ichneumonidae se reflejó en primavera y verano; durante el invierno se presentó la menor diversidad (tabla 2), a diferencia de lo documentado por Rodríguez-Vélez, Zaragoza-Caballero y Rodríguez (2009) en Oaxaca para Encyrtidae y otras familias de Hymenoptera, en donde la diversidad mayor se observó en febrero.

Existe una correlación positiva entre la temperatura ambiental del área de estudio y la diversidad de Ichneumonidae (fig. 4): a mayor temperatura, mayor diversidad de especies de icneumónidos, situación que no se presentó en relación con la precipitación 
pluvial registrada, ya que la correlación existente fue moderada (fig. 3). Estos resultados difieren con lo documentado por González-Moreno y Bordera (2011) para Ichneumonidae en Yucatán, en donde las recolectas fueron más abundantes durante la temporada de lluvias. Sin embargo, bajo las condiciones de este estudio, esta familia presentó mayor abundancia en un rango de temperatura media de $17.6-21.8^{\circ} \mathrm{C}$ (fig. 2).

Por lo anterior, se puede concluir que la diversidad de Ichneumonidae en el ejido Magdaleno Aguilar de Jaumave es alta. La temperatura ambiental estuvo correlacionada con la diversidad de Ichneumonidae de forma positiva. La precipitación pluvial presentó una correlación moderada con la diversidad de Ichneumonidae. Los nuevos registros de Ichneumonidae para México de Diapetimorpha rufigaster, Dichrogaster gemina y Poemenia albipes aumentan la cifra de 1,295
(Castillo-Flores, Bordera, Ruíz-Cancino y Coronado-Blanco, 2014; González-Moreno y Bordera, 2011; Ruíz-Cancino, Khalaim, Coronado-Blanco, Toledo-Hernández y CoronaLópez, 2014a y Ruíz-Cancino, Rafaelevich-Kasparyan, González-Moreno, Khalaim y Coronado-Blanco, 2014b) a 1,298 especies de Ichneumonidae para el país.

\section{Agradecimientos}

Al proyecto «Taxonomía y ecología de fauna y micobiota en comunidades forestales y cultivos», de la red de CA de PROMEP, y a la UAT, por su apoyo para esta investigación.

Anexo 1. Ichneumonidae recolectados durante un año mediante una trampa Malaise en Jaumave, Tamaulipas, México (septiembre de 2011-agosto de 2012).

\begin{tabular}{|c|c|c|c|c|c|c|c|c|c|c|c|c|c|c|c|}
\hline \multirow{2}{*}{$\begin{array}{l}\text { Subfamilia } \\
\text { Género y/o especie }\end{array}$} & \multicolumn{13}{|c|}{ Meses } & \multicolumn{2}{|c|}{ Sexo } \\
\hline & sep & oct & nov & dic & ene & feb & mar & abr & may & jun & jul & ago & Total & $\sigma^{7}$ & q \\
\hline \multicolumn{16}{|l|}{ Alomyinae } \\
\hline Centeterus sp. 2 & & 1 & & 1 & & & & & & & & & 2 & 1 & 1 \\
\hline Centeterus sp. 3 & & & & & & & & & & & 2 & & 2 & 2 & 0 \\
\hline \multicolumn{16}{|l|}{ Anomaloninae } \\
\hline Diradops hyphantriae Kasparyan y & & & & & & & & & & & & 2 & 2 & 1 & 1 \\
\hline \multicolumn{16}{|l|}{ Pinson, 2007} \\
\hline Diradops sp. & & & & & & & & & & 1 & & & 1 & 1 & 0 \\
\hline Exetastes pictus Cushman & 1 & 1 & & & & & & & 4 & 8 & 3 & 3 & 20 & 18 & 2 \\
\hline 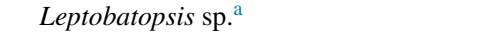 & & & & & & & & & & 1 & & & 1 & 1 & 0 \\
\hline Lissonota sp. 1 & & & & & & & & 1 & & & & & 1 & 1 & 0 \\
\hline Meniscomorpha sp. 1 & & & & & & & & & & & & 1 & 1 & 0 & 1 \\
\hline Meniscomorpha sp. 2 & & & & & & & & & 1 & & & & 1 & 0 & 1 \\
\hline Meniscomorpha sp. 3 & & 1 & & & & & & & & & & & 1 & 1 & 0 \\
\hline Meniscomorpha sp. 4 & & 1 & & & & & & & & & & & 1 & 1 & 0 \\
\hline Meniscomorpha sp. 5 & & & & & & & & & & & & 1 & 1 & 0 & 1 \\
\hline Meniscomorpha sp. 6 & & & & & & & & & 1 & & & & 1 & 0 & 1 \\
\hline \multicolumn{16}{|l|}{ Brachycyrtinae } \\
\hline Brachycyrtus pretiosus Cushman, 1936 & & & & & & & & & 2 & & 1 & & 3 & 0 & 3 \\
\hline \multicolumn{16}{|l|}{ Campopleginae } \\
\hline Campoctonus sp. & & 2 & & & & & & & & 1 & & & 3 & 1 & 2 \\
\hline Campoletis sp. 1 & & & & & & & & & 2 & 1 & & & 3 & 2 & 1 \\
\hline Campoplex sp. 6 & & & & & & & & & & & & 1 & 1 & 0 & 1 \\
\hline Casinaria sp. 1 & & & & & & & 1 & 2 & 5 & 3 & & & 11 & 0 & 11 \\
\hline Casinaria sp. 2 & & & & & & & 1 & 1 & 4 & 6 & 1 & & 13 & 6 & 7 \\
\hline
\end{tabular}


Anexo 1 (Continuación)

\begin{tabular}{|c|c|c|c|c|c|c|c|c|c|c|c|c|c|c|c|}
\hline \multirow{2}{*}{$\begin{array}{l}\text { Subfamilia } \\
\text { Género y/o especie }\end{array}$} & \multicolumn{13}{|c|}{ Meses } & \multicolumn{2}{|c|}{ Sexo } \\
\hline & sep & oct & nov & dic & ene & feb & mar & $\mathrm{abr}$ & may & jun & jul & ago & Total & $0^{7}$ & 우 \\
\hline Casinaria sp. 3 & & & & & & & & & & & & 1 & 1 & 0 & 1 \\
\hline Casinaria sp. 4 & & & & 1 & & & & & & & & & 1 & 0 & 1 \\
\hline Cymodusa sp. 1 & & & 1 & & & 1 & 1 & 1 & & & & & 4 & 0 & 4 \\
\hline Cymodusa sp. 2 & & & & & & & & & 1 & & & & 1 & 0 & 1 \\
\hline Diadegma sp. 1 & 3 & 6 & & & 5 & 2 & 3 & 8 & 12 & 3 & 1 & 1 & 44 & 21 & 23 \\
\hline Diadegma sp. 2 & & & & & & & & 2 & & & & & 2 & 1 & 1 \\
\hline Diadegma sp. 3 & & & & & & & & 1 & & & & & 1 & 0 & 1 \\
\hline Diadegma sp. 4 & & & & & & & & & 1 & & & & 1 & 1 & 0 \\
\hline Diadegma sp. 5 & & 1 & & & & & & 4 & 1 & 1 & & & 7 & 2 & 5 \\
\hline Diadegma sp. 6 & & & & & & & & 1 & & & & & 1 & 0 & 1 \\
\hline Diadegma sp. 7 & & & & & & & & 1 & & & & & 1 & 0 & 1 \\
\hline Diadegma sp. 8 & 1 & & 6 & & 5 & 3 & 14 & 13 & 7 & 2 & 2 & 5 & 58 & 26 & 32 \\
\hline Diadegma sp. 9 & & & & & & & & 6 & 2 & & & & 8 & 2 & 6 \\
\hline Diadegma sp. 10 & & & & & & & & & & & & 1 & 1 & 0 & 1 \\
\hline Dusona sp. 1 & & & & & 1 & & 1 & 1 & 1 & & 3 & & 7 & 3 & 4 \\
\hline Dusona sp. 2 & & & & & & & & & & & 1 & 1 & 2 & 1 & 1 \\
\hline Hyposoter sp. 1 & 1 & 1 & & & 2 & & 1 & 1 & 2 & 1 & & & 9 & 3 & 6 \\
\hline Hyposoter sp. 2 & & & & & & & & & 1 & & & & 1 & 0 & 1 \\
\hline Hyposoter sp. 3 & & & & & & & & & 1 & & & & 1 & 0 & 1 \\
\hline Hyposoter sp. 4 & & 2 & & 1 & & 1 & 1 & 10 & 7 & 4 & & 1 & 27 & 6 & 21 \\
\hline Hyposoter sp. 5 & & & & & & & & & & 4 & 2 & 3 & 9 & 2 & 7 \\
\hline Hyposoter sp. 6 & & & & & & & & 4 & 4 & & 1 & & 9 & 4 & 5 \\
\hline Hyposoter sp. 7 & & 1 & & & & & & & & 2 & & 1 & 4 & 2 & 2 \\
\hline Microcharops sp. & 2 & & & & & & 2 & 1 & 9 & 3 & 3 & 2 & 22 & 7 & 15 \\
\hline Phobocampe sp. 1 & & & & & & & & 2 & 2 & & & & 4 & 2 & 2 \\
\hline Phobocampe sp. 2 & & & & & & & & & & & & 1 & 1 & 1 & 0 \\
\hline Phobocampe sp. 3 & & & & & & & & & & 2 & 1 & & 3 & 2 & 1 \\
\hline Phobocampe sp. 4 & & & & & & & & 1 & 2 & & 1 & 3 & 7 & 7 & 0 \\
\hline Venturia sp. 1 & & & & & & & & & & & & 1 & 1 & 0 & 1 \\
\hline Venturia sp. 2 & 1 & & & & & & & & & & & & 1 & 0 & 1 \\
\hline \multicolumn{16}{|l|}{ Cryptinae } \\
\hline $\begin{array}{l}\text { Acerastes bimaculator Kasparyan y Ruíz, } \\
2008\end{array}$ & & & & & & & & & & 1 & 1 & 6 & 8 & 5 & 3 \\
\hline A. myartsevae Kasparyan y Ruíz, 2008 & & & & & & & & & & & 1 & & 1 & 1 & 0 \\
\hline A. tinctor Kasparyan y Ruíz, 2008 & & & & & & & & & & & 6 & 6 & 12 & 8 & 4 \\
\hline Acrolyta sp. & & & 1 & & & & & & & & & & 1 & 0 & 1 \\
\hline $\begin{array}{l}\text { Agonocryptus chichimecus (Cresson, } \\
\text { 1874) }\end{array}$ & & & 1 & & & & & & 2 & 2 & 1 & 2 & 8 & 1 & 7 \\
\hline $\begin{array}{l}\text { Bathyzonus interruptor Kasparyan y Ruíz, } \\
2008\end{array}$ & & & & & & & & & & & & 1 & 1 & 1 & 0 \\
\hline Bicryptella sp. & & 1 & & & & & & & & & & & 1 & 1 & 0 \\
\hline $\begin{array}{l}\text { Cestrus arcuatorius Kasparyan y Ruíz, } \\
2005\end{array}$ & & & & & & & & & & 1 & & & 1 & 1 & 0 \\
\hline Cestrus sp. & & & & & & & & 1 & & & & & 1 & 1 & 0 \\
\hline $\begin{array}{l}\text { Compsocryptus xanthostigma (Brullé, } \\
\text { 1846) }\end{array}$ & & 1 & & & & & & & 1 & 4 & 2 & & 8 & 8 & 0 \\
\hline Cryptus albitarsis (Cresson, 1864) & & & 1 & & & & & & & & 5 & 3 & 9 & 8 & 1 \\
\hline C. rugifrons (Townes, 1962) & & & & & & & & & & & 1 & 1 & 2 & 0 & 2 \\
\hline C. scapulatus (Townes, 1962) & & & & & & & & & 1 & & & & 1 & 0 & 1 \\
\hline C. sp. 1 & & & & & & & & & 1 & & & & 1 & 0 & 1 \\
\hline C. sp. 2 & & & & & & & & & & & & 1 & 1 & 0 & 1 \\
\hline Diaglyptidea sp. & 1 & 3 & 2 & & 1 & 1 & & 4 & 2 & 1 & 1 & & 16 & 4 & 12 \\
\hline Diapetimorpha aspila Porter, 1977 & & & & & & & 1 & & 1 & & & 2 & 4 & 4 & 0 \\
\hline $\begin{array}{l}\text { Diapetimorpha delfini Kasparyan y Ruíz, } \\
2005\end{array}$ & & & & & & & & & & & 1 & & 1 & 1 & 0 \\
\hline $\begin{array}{l}\text { Diapetimorpha pronotalis Kasparyan y } \\
\text { Ruíz, } 2005\end{array}$ & & & & & & & & & & & 1 & & 1 & 0 & 1 \\
\hline $\begin{array}{l}\text { Diapetimorpha rufigaster }{ }^{\mathrm{b}} \text { Cushman, } \\
1929\end{array}$ & & & & & & & & & & 1 & & & 1 & 1 & 0 \\
\hline Diapetimorpha sp. & & & & & & & & & & 1 & 5 & 1 & 7 & 7 & 0 \\
\hline Dichrogaster chrysopae (Ashmead, 1894) & 1 & 1 & & & 2 & & 1 & & & & & & 5 & 5 & 0 \\
\hline D. gemina ${ }^{\mathrm{b}}$ & & 1 & 1 & 1 & 1 & & 1 & 3 & 2 & 1 & 2 & 2 & 15 & 0 & 15 \\
\hline
\end{tabular}


Anexo 1 (Continuación)

\begin{tabular}{|c|c|c|c|c|c|c|c|c|c|c|c|c|c|c|c|}
\hline \multirow{2}{*}{$\begin{array}{l}\text { Subfamilia } \\
\text { Género y/o especie }\end{array}$} & \multicolumn{13}{|c|}{ Meses } & \multicolumn{2}{|c|}{ Sexo } \\
\hline & sep & oct & nov & dic & ene & feb & mar & abr & may & jun & jul & ago & Total & $\sigma^{7}$ & 우 \\
\hline D. n. sp. & & & 3 & & 1 & & 2 & 3 & 2 & 1 & 1 & & 13 & 0 & 13 \\
\hline Epelaspis sp. 1 & 1 & 2 & 2 & & & & & & & & & & 5 & 3 & 2 \\
\hline Epelaspis sp. 2 & 1 & & & & & & & & & & & & 1 & 0 & 1 \\
\hline $\begin{array}{l}\text { Epicnemion lineator Kasparyan y Ruíz, } \\
2008\end{array}$ & & & & & & & & & & & 2 & & 2 & 1 & 1 \\
\hline Ethelurgus sp. & & 1 & & & & & & & & & & & 1 & 0 & 1 \\
\hline Gelis sp. & 1 & & & & & & 1 & 3 & 1 & 2 & & 2 & 10 & 10 & 0 \\
\hline $\begin{array}{l}\text { Ischnus inquisitorius atricollaris (Walsh, } \\
1873 \text { ) }\end{array}$ & & & & & & & & & & & & 1 & 1 & 0 & 1 \\
\hline Ischnus ?laevifrons Townes, 1962 & & 1 & & & & & & & & & & & 1 & 1 & 0 \\
\hline Ischnus sp. 1 & & 1 & & & & & & 1 & 1 & & & & 3 & 0 & 3 \\
\hline Ischnus sp. 2 & & 1 & & & & & & & 1 & & & & 2 & 0 & 2 \\
\hline Joppidium ardens Cresson, 1874 & & & & & & & & & 1 & & & & 1 & 0 & 1 \\
\hline J. brochum Townes, 1962 & & & & & & & & & 2 & 2 & & & 4 & 4 & 0 \\
\hline Lanugo hebetis (Cameron, 1885) & & & & & & & & & & 1 & & 1 & 2 & 2 & 0 \\
\hline L. picta Townes, 1962 & & 1 & & & & & & 1 & 7 & 2 & & & 11 & 6 & 5 \\
\hline Lymeon ?cinctiventris (Cushman, 1929) & & & & & & & & & & 1 & & 4 & 5 & 5 & 0 \\
\hline L. moratus (Cresson, 1874) & & & & & & & & & & 1 & 1 & 1 & 3 & 2 & 1 \\
\hline L. tricoloripes Kasparyan y Ruíz, 2004 & & & & & & & & & & & & 1 & 1 & 1 & 0 \\
\hline Lymeon sp. 1 & 1 & & & & & & & & 1 & 2 & 1 & 3 & 8 & 1 & 7 \\
\hline Lymeon sp. 2 & & & & & & & & & & 1 & & & 1 & 1 & 0 \\
\hline Mesostenus gracilis Cresson, 1864 & & 1 & & & & & & & & & & & 1 & 0 & 1 \\
\hline M. madronio Kasparyan y Ruíz, 2008 & 3 & 1 & 1 & & 1 & & & & 3 & & & 1 & 10 & 4 & 6 \\
\hline M. pertenius Cresson, 1874 & & & & & & & & 1 & & & 1 & & 2 & 1 & 1 \\
\hline M. thoracicus Cresson, 1864 & & & & & & & & & & & & 1 & 1 & 1 & 0 \\
\hline $\begin{array}{l}\text { Messatoporus compressicornis Cushman, } \\
1929\end{array}$ & & & & & & & & 1 & & 3 & 3 & & 7 & 1 & 6 \\
\hline M. discoidalis (Cresson, 1872) & & & & & & & & & & 2 & 1 & 1 & 4 & 2 & 2 \\
\hline M. terebrator Kasparyan y Ruíz, 2005 & & & & & & & & & 3 & & & 1 & 4 & 2 & 2 \\
\hline M. tibiator Kasparyan y Ruíz, 2005 & & & & & & & & & & & 1 & 1 & 2 & 1 & 1 \\
\hline Orthizema sp. & & & & & & 1 & & & & & & & 1 & 0 & 1 \\
\hline Pachysomoides stupidus (Cresson, 1874) & 2 & & & & & & & 3 & 4 & 3 & 10 & 9 & 31 & 0 & 31 \\
\hline Toechorychus sp. & & & & & & & & & & 1 & 3 & 1 & 5 & 0 & 5 \\
\hline Xenolytus sp. & 1 & & & & & & & & & & & & 1 & 0 & 1 \\
\hline \multicolumn{16}{|l|}{ Diplazontinae } \\
\hline Diplazon sp. & & 1 & & & & & 1 & & & & & & 2 & 1 & 1 \\
\hline \multicolumn{16}{|l|}{ Ichneumoninae } \\
\hline ?Barichneumon sp. 1 & & & & & 1 & & 1 & 2 & 1 & & & 3 & 8 & 8 & 0 \\
\hline ?Barichneumon sp. 2 & 1 & & & & & & & & & & & & 1 & 1 & 0 \\
\hline Carinodes sp. & & & & & & & & 1 & & & & 1 & 2 & 1 & 1 \\
\hline Coelichneumon sp. & & & & 1 & & & & & 1 & & & 2 & 4 & 2 & 2 \\
\hline Dicaelotus sp. & & & & & & & & 1 & 1 & & & & 2 & 1 & 1 \\
\hline Dilopharius sp. & & 1 & & & & & & & & & & 2 & 3 & 2 & 1 \\
\hline Eurydacus sp. & & & & & & & & & 3 & & & & 3 & 0 & 3 \\
\hline Limonethe sp. 1 & & & & & & & & 1 & 1 & & & 2 & 4 & 3 & 1 \\
\hline Limonethe sp. 2 & & & & & & & & & & & 2 & 2 & 4 & 2 & 2 \\
\hline Limonethe sp. 3 & 1 & 1 & & & & & & & 1 & & & 1 & 4 & 0 & 4 \\
\hline Lophojoppa sp. & & & & & & & & & & & 1 & & 1 & 1 & 0 \\
\hline Narthecura sp. & 1 & & & & & & & & & & & 1 & 2 & 0 & 2 \\
\hline Oedicephalus sp. & & 1 & & & & & & & & & & & 1 & 1 & 0 \\
\hline Oezdemirus sp. 1 & & & & & & & & 1 & & & & & 1 & 0 & 1 \\
\hline Oezdemirus sp. 2 & & & & & & & & & & & & 1 & 1 & 0 & 1 \\
\hline Patroclus sp. & & & & & & & & & & & & 7 & 7 & 7 & 0 \\
\hline Phaeogenes sp. & & & & & & & & & & & 2 & & 2 & 2 & 0 \\
\hline Plagiotrypes sp. & & & & & & & & & & 1 & & & 1 & 1 & 0 \\
\hline Projoppa sp. & & & & & & & & 1 & & & & & 1 & 0 & 1 \\
\hline Stenichneumon sp. & 1 & & 1 & & & & 2 & 2 & 2 & & 4 & & 12 & 3 & 9 \\
\hline Trogomorpha sp. & 1 & 1 & 1 & & & & & & 1 & & & & 4 & 1 & 3 \\
\hline \multicolumn{16}{|l|}{ Mesochorinae } \\
\hline Mesochorus sp. 1 & & & & & & & & & & & & 1 & 1 & 0 & 1 \\
\hline Mesochorus sp. 2 & & 1 & & & & & & & & 1 & & 1 & 3 & 0 & 3 \\
\hline Mesochorus sp. 3 & & 2 & 1 & & 1 & & & & & & & 1 & 5 & 0 & 5 \\
\hline Mesochorus sp. 4 & & & & & & & & & & & 1 & & 1 & 0 & 1 \\
\hline
\end{tabular}


Anexo 1 (Continuación)

\begin{tabular}{|c|c|c|c|c|c|c|c|c|c|c|c|c|c|c|c|}
\hline \multirow{2}{*}{$\begin{array}{l}\text { Subfamilia } \\
\text { Género y/o especie }\end{array}$} & \multicolumn{13}{|c|}{ Meses } & \multicolumn{2}{|c|}{ Sexo } \\
\hline & sep & oct & nov & dic & ene & feb & mar & abr & may & jun & jul & ago & Total & $\sigma^{7}$ & 우 \\
\hline \multicolumn{16}{|l|}{ Metopiinae } \\
\hline $\begin{array}{l}\text { Hypsicera cuneata Townes y Townes, } \\
1959\end{array}$ & & & 1 & & 1 & 1 & 2 & 2 & 6 & & & 1 & 14 & 0 & 14 \\
\hline Hypsicera sp. 1 & & & & & & 1 & & & & & & & 1 & 0 & 1 \\
\hline Triclistus sp. & & 1 & & & & & & & & & & & 1 & 1 & 0 \\
\hline \multicolumn{16}{|l|}{ Nonninae } \\
\hline Nonnus sp. & & & 1 & & & & & & 1 & & & 1 & 3 & 2 & 1 \\
\hline \multicolumn{16}{|l|}{ Ophioninae } \\
\hline Enicospilus sp. 1 & & & & & & & & & 1 & & & & 1 & 0 & 1 \\
\hline Enicospilus sp. 2 & & & & & & & & & & 1 & & & 1 & 0 & 1 \\
\hline \multicolumn{16}{|l|}{ Orthocentrinae } \\
\hline Chilocyrtus sp. & & & & & & & & 1 & & & & & 1 & 0 & 1 \\
\hline Orthocentrus sp. & & & 1 & & & & 1 & 18 & 15 & 6 & 22 & 9 & 72 & 8 & 64 \\
\hline Stenomacrus sp. & & & & & & & & & 1 & & & & 1 & 1 & 0 \\
\hline \multicolumn{16}{|l|}{ Pimplinae } \\
\hline Acrotaphus tibialis (Cameron, 1886) & & & & & & & & & & & & 2 & 2 & 1 & 1 \\
\hline 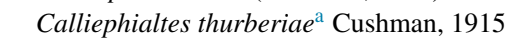 & & & & & & & & & 1 & 1 & & 1 & 3 & 0 & 3 \\
\hline Clistopyga ?fernandezi Gauld, 1991 & & & & & & & & & & & & 1 & 1 & 1 & 0 \\
\hline C. recurva $($ Say, 1835$)$ & & & & & & & & 1 & & & & & 1 & 0 & 1 \\
\hline Iseropus $\mathrm{sp} .1$ & & 1 & & & & & & & & & & 2 & 3 & 3 & 0 \\
\hline Iseropus $\mathrm{sp} .2$ & & & & & & & & & & & & 1 & 1 & 1 & 0 \\
\hline Itoplectis ?marianneae Gauld, 1991 & & & & & & & & & & & & 1 & 1 & 1 & 0 \\
\hline Neotheronia concolor Krieger, 1905 & & & & & & & & & & & 1 & 1 & 2 & 1 & 1 \\
\hline N. mellosa (Cresson, 1874) & & & & & & & & & & & 1 & 1 & 2 & 1 & 1 \\
\hline N. tacubaya (Cresson, 1874) & & & & & & & & & & & & 1 & 1 & 0 & 1 \\
\hline Pimpla sanguinipes Cresson, 1872 & 2 & & & & & & & & & & & & 2 & 1 & 1 \\
\hline Pimpla spp. (2) & 5 & 9 & 8 & 5 & 12 & 3 & 9 & 37 & 78 & 24 & 32 & 43 & 265 & 204 & 61 \\
\hline Polysphincta ?dizardi Gauld, 1991 & & & & & & & & & & & & 2 & 2 & 1 & 1 \\
\hline P. ?mascoi Gauld, 1991 & & & & & & & 1 & & & & & & 1 & 0 & 1 \\
\hline Scambus basseyi Gauld, 1991 & & & & & & & & & & & & 2 & 2 & 1 & 1 \\
\hline Scambus sp. & & & 1 & & & & & & & & & & 1 & 1 & 0 \\
\hline Tromatobia blancoi Gauld, 1991 & & & & & & & & & & & 2 & & 2 & 1 & 1 \\
\hline T. ?rufopectus & & & & & & & & & & 1 & & & 1 & 0 & 1 \\
\hline Tromatobia sp. & & & & & & & & 1 & & 1 & 2 & & 4 & 0 & 4 \\
\hline Zaglyptus simonis Marshall, 1892 & & & & & & & & 4 & 9 & 2 & 3 & & 18 & 1 & 17 \\
\hline Zatypota alborhombarta (Davis, 1895) & & & & & & & 1 & & & 2 & 1 & & 4 & 1 & 3 \\
\hline \multicolumn{16}{|l|}{ Poemeninae } \\
\hline Poemenia albipes ${ }^{\mathrm{b}}$ Cresson, 1870 & & & & & & & & & 1 & & & & 1 & 0 & 1 \\
\hline \multicolumn{16}{|l|}{ Tersilochinae } \\
\hline Allophrys divaricata Horstmann, 2010 & & & & & & & & & & 1 & & & 1 & 0 & 1 \\
\hline Nuevo género & & & & & & & & & 1 & & & & 1 & 1 & 0 \\
\hline Tryphoninae & & & & & & & & & & & & & & & \\
\hline Netelia sp. & & 1 & & & & & 2 & & & & & & 3 & 0 & 3 \\
\hline Total & 36 & 54 & 36 & 11 & 36 & 14 & 56 & 161 & 254 & 129 & 149 & 179 & 1,115 & 524 & 591 \\
\hline
\end{tabular}

${ }^{a}$ Nuevo registro para Tamaulipas.

b Nuevo registro para México.

\section{Referencias}

Anento, J. L. y Selfa, J. (1997). Himenópteros Parasitica y control de plagas Boletín de la Sociedad Entomológica Aragonesa, 20, 151-160.

Castillo-Flores, P. M., Bordera, S., Ruíz-Cancino, E. y Coronado-Blanco, J. M. (2014). Nuevos registros para México de tres especies de Endasys Foerster, 1869 (Hymenoptera: Ichneumonidae). Acta Zoológica Mexicana, 30, $723-726$.

Falcó, G. J. V., Oltra, M. M. T., Moreno, M. J., Pujade-Villar, J. y Jiménez, P. R. (2006). Fenología de los bracónidos (Hymenoptera: Ichneumonoidea, Braconidae) del Pirineo Andorrano. Pirineos, 161, 111-132.

Gauld, I. D. (1991). The Ichneumonidae of Costa Rica, 1. Memoirs of the American Entomological Institute, 47, 1-589.
Gauld, I. D. (2006). Familia Ichneumonidae. En P. E. Hanson y I. D. Gauld (Eds.), Hymenoptera de la Región Neotropical (pp. 446-487). Gainesville: Memoirs of the American Entomological Institute.

Gauld, I. D. y Bolton, B. (1988). The Hymenoptera. Londres: British Museum (Natural History)

Gauld, I. D., Godoy, C., Sithole, R. y Ugalde, G. J. (2002). The Ichneumonidae of Costa Rica, 4. Memoirs of the American Entomological Institute, 66, 1-768.

Gauld, I. D., Wahl, D., Bradshaw, K., Hanson, P. y Ward, S. (1997). The Ichneumonidae of Costa Rica, 2. Memoirs of the American Entomological Institute, $57,1-485$.

Gauld, I. D., Ward, S. y Mallet, V. (2000). The Ichneumonidae of Costa Rica, 3. Memoirs of the American Entomological Institute, 63, $1-453$. 
González-Moreno, A. y Bordera, S. (2011). New records of Ichneumonidae (Hymenoptera: Ichneumonoidea) from Mexico. Zootaxa, 2879, 1-21.

Halffer, G. y Moreno, C. E. (2005). Significado biológico de las diversidades alfa, beta y gamma. En G. Halffer, J. Soberón, P. Koleff y A. Melic (Eds.), Sobre diversidad biológica: el significado de las diversidades alfa, beta y gamma (pp. 5-18). Zaragoza: m3m-Monografías Tercer Milenio.

Hopkins, K. D., Hopkins, B. R. y Glass, G. V. (1997). Estadística básica para las ciencias sociales y del comportamiento. México, D. F: Prentice-Hall Hispanoamericana.

Kasparyan, D. R. y Ruíz, C. E. (2005). Cryptini de México (Hymenoptera: Ichneumonidae: Cryptinae), Parte I. Avispas parasíticas de plagas y otros insectos. Cd. Victoria: Universidad Autónoma de Tamaulipas.

Kasparyan, D. R. y Ruíz, C. E. (2008). Cryptini de México (Hymenoptera: Ichneumonidae: Cryptinae), Parte II. Serie Avispas parasíticas de plagas y otros insectos. Cd. Victoria: Universidad Autónoma de Tamaulipas.

Khalaim, A. I. y Ruíz-Cancino, E. (2012). Mexican species of Exetastes (Hymenoptera: Ichneumonidae: Banchinae), with description of three new species. Revista Mexicana de Biodiversidad, 83, 370-379.

Moreno, C. E. (2001). Métodos para medir la biodiversidad. Zaragoza: CYTED, ORCYT/UNESCO y SEA.

Nicholls, E. C. I. (2008). Control biológico de insectos: un enfoque agroecológico. Antioquia: Editorial Universidad de Antioquia

Pérez-Urbina, B., Correa-Sandoval, A., Ruíz-Cancino, E., Kasparyan, D. R., Coronado-Blanco, J. M. y Horta-Vega, J. V. (2010). Diversidad de Ichneumonidae (Hymenoptera) en el Cañón del Novillo, Victoria, Tamaulipas, México. Entomotropica, 25, 83-97.

Rodríguez-Vélez, B., Zaragoza-Caballero, S. y Rodríguez, J. M. (2009). Diversidad de Encyrtidae (Hymenoptera: Chalcidoidea) y otras familias de Hymenoptera obtenidas con trampa Malaise en el bosque tropical caducifolio de la región de Huatulco, Oaxaca, México. Revista Mexicana de Biodiversidad, 80, 709-719.

Ruíz, C. E. (2010). Ichneumonidae (Hymenoptera) del estado de Tamaulipas, México. Serie de avispas parasíticas de plagas y otros insectos No. 6. México, D. F: Editorial Planea.
Ruíz-Cancino, E., Khalaim, A. I., Coronado-Blanco, J. M., Toledo-Hernández, V. H. y Corona-López, A. M. (2014). Registro de Diradops bionica Ugalde y Gauld, 2002 (Hymenoptera: Ichneumonidae: Banchinae) para México y de otras dos especies para Oaxaca. Acta Zoológica Mexicana, 30, 427-429.

Ruíz-Cancino, E., Rafaelevich-Kasparyan, D., González-Moreno, A., Khalaim, A. I. y Coronado-Blanco, J. M. (2014). Biodiversidad de Ichneumonidae (Hymenoptera) en México. Revista Mexicana de Biodiversidad, 85 385-391.

Sulca-Garro LA, Castillo C (2013). Distribución de Rogadinae (Hymenoptera: Braconidae) en un gradiente altitudinal en los Andes del sur de Perú. II Encuentro de Investigadores. Ministerio del Ambiente. Perú [consultado 27 May 2015]. Disponible en: http://es.slideshare.net/InfoAndina/presentacion-24300723.

Townes, H. K. (1969). The genera of Ichneumonidae. Part 1. Memoirs of the American Entomological Institute, 11, 1-300.

Townes, H. K. (1970a). The genera of Ichneumonidae. Part 2. Memoirs of the American Entomological Institute, 12, 1-537.

Townes, H. K. (1970b). The genera of Ichneumonidae. Part 3. Memoirs of the American Entomological Institute, 13, 1-307.

Townes, H. K. (1971). The genera of Ichneumonidae. Part 4. Memoirs of the American Entomological Institute, 17, 1-372.

Townes, H. K. y Townes, M. (1959). Ichneumon-Flies of American North of Mexico: 1. Subfamily Metopiinae. Washington DC: United States National Museum Bulletin 216. Smithsonian Institution.

Townes, H. K. y Townes, M. (1966). A catalog and reclassification of the Neotropical Ichneumonidae. Memoirs of the American Entomological Institute, $8,1-366$.

Townes, H. K. y Townes, M. (1978). Ichneumon-flies of American North of Mexico: 7. Subfamily Banchinae, tribes Lissonotini and Banchini. Memoirs of the American Entomological Institute, 26, 1-614.

Whittaker, R. H. (1960). Vegetation of the Siskiyou Mountains, Oregon and California. Ecological Monographs, 30, 279-338.

Yu, D. S., van Achterberg, C. y Horstman, K. (2012). World Ichneumonoidea 2011. Taxonomy, Biology, Morphology and Distribution. Ottawa: Taxapad. 